\title{
Some influences of remote topography on western boundary currents
}

\author{
by Michael A. Spall ${ }^{1}$
}

\begin{abstract}
The influence of topography in a basin interior on the separation and time-dependence of strongly nonlinear western boundary currents is explored using a shallow water numerical model and scaling theory. In the linear limit, the western boundary current follows the western boundary to the latitude of the gap in the interior topography where it then separates from the coast and flows eastward in a narrow jet. As nonlinearity is increased, the flow initially remains steady but develops a series of stationary meanders extending off the western boundary at the separation latitude. For strongly nonlinear flows the solutions become time-dependent. The mean separation latitude continues to be tied to the interior topography even though the mean zonal flow far exceeds the baroclinic wave speed. In most cases, the variability is dominated by westward-propagating cyclonic and anticyclonic meanders of the separated western boundary current. The behavior on the western boundary alternates between an overshoot of the western boundary current with an anticyclonic meander and a premature separation of the western boundary current with the poleward formation of an anticyclonic eddy. The mean flow is consistent with the separation latitude of the North Atlatic Current to the west of the Charlie Gibbs Fracture Zone and the time-dependence shows many similarities with the observed variability of the East Australian Current to the west of New Zealand. The wavelength of the meanders and the frequency and amplitude of the oscillations are well predicted by a simple scaling that accounts for wave propagation, nonlinear advection, and a viscous sublayer along the western boundary.
\end{abstract}

\section{Introduction}

Topography in the form of tall ridges or islands can exert a strong influence on the largescale ocean circulation. Zonal flows are blocked by such features and must pass through gaps in ridges or around the tips of islands to connect the circulation between adjacent ocean basins. In the linear limit, the influence of these topographic features extends infinitely far to the west (although it will be modified by friction), with Rossby waves eventually delivering their message to the western boundary. However, for sufficiently strong eastward flows advection can overwhelm the baroclinic wave speed and propagate information eastwards. In such cases, it is not obvious how the influences of waves and nonlinear advection conspire

1. Department of Physical Oceanography, Woods Hole Oceanograhic Institution. Address for correspondence: MS\#21, 360 Woods Hole Road, Woods Hole, MA 02543.e-mail: mspall@whoi.edu 
to close the circulation between a separated western boundary current and a topographic feature in the ocean interior. Some of these issues also arise in simply connected closed domains, but topography is used here as a means to permit strong zonal flows all across the basin and to generate pressure anomalies on the eastern boundary.

The phenomenology explored in this study falls into the broad category of the transition from linear, steady solutions to time-dependent periodic solutions and eventually to chaotic solutions. Such transitions are commonly found as nonlinearity of a system increases. Oceanographic examples include wind-driven circulation (Jiang et al., 1995), two-dimensional thermohaline circulation (Dijkstra and Molemaker, 1997), and ENSO (Dijkstra and Neelin, 1995). A nice review of the dynamical systems approach to studying such transitions can be found in Dijkstra and Ghil (2005). These studies are often based on idealized, relatively low-order systems, yet the basic behavior is often found in more complex and dynamically complete models. It is likely that the transitions discussed here can also be described in terms of similar Hopf bifurcations.

There are many examples of strong western boundary current flows that separate from the boundary and flow eastward in regions to the west of significant topogaphic features. The North Atlantic Current separates from the western boundary near $50^{\circ} \mathrm{N}$, roughly at the latitude of the Charlie Gibbs Fracture Zone, a prominant gap in the Mid-Atlantic Ridge that lies approximately $1000 \mathrm{~km}$ to the east (Bower and von Appen, 2008, Woityra and Rossby, 2008). Analysis of float data shows that the North Atlantic Current preferentially flows over deep gaps in the ridge system even though the topography does not extend into the upper ocean (Bower and von Appen, 2008). In the South Atlantic, the East Australian Current separates from the coast of Australia and flows eastward in the Tasman Front, eventually passing around the northern tip of New Zealand approximately $1000 \mathrm{~km}$ to the east (Nilsson and Cresswell, 1981). The separation point and the frontal region associated with the current are both highly nonlinear and time-dependent. In the North Pacific, the Shatsky Rise is a large plateau approximately $1500 \mathrm{~km}$ east of the Kuroshio separation point. In the Southern Ocean, the Antarctic Circumpolar Current follows the western boundary northwards just downstream of the Drake Passage before turning to the east. While the Antarctic Circumpolar Current separates from the South American continent approximately $6000 \mathrm{~km}$ to the west of the Kerguelen Plateau, linear wind-driven models suggest that this topographic feature controls the separation point downstream of the Drake Passage (LaCasce and Isachsen, 2010). While each of these situations differ, they provide examples of highly nonlinear flows near a western boundary with some indication that they are influenced by topography located far to the east.

The above examples motivate a very basic question: how do topographic features in the basin interior influence and interact with flows near the western boundary? For example, in the nonlinear regime, does the influence of topography still get propagated infinitely far to the west by Rossby waves? If there is a nonlinear flow through a narrow gap in topography, how far to the east can such a flow penetrate? In other words, how far to the east and west of a gap in topography is the nonlinear ocean circulation influenced by that 


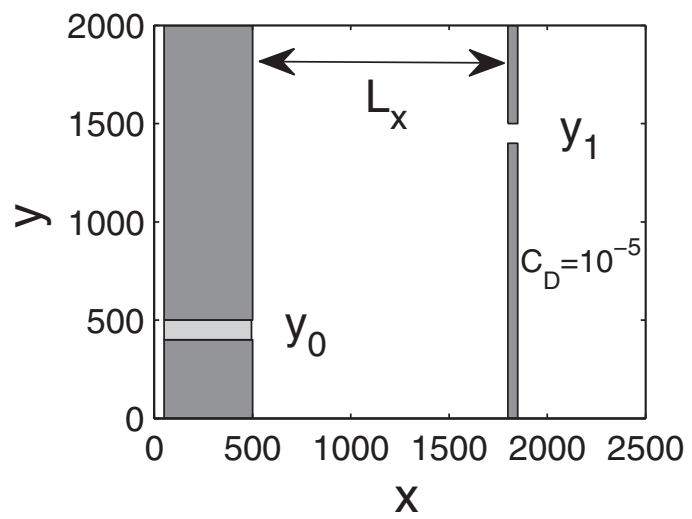

Figure 1. Model domain with dark shading for boundaries and light shading for the forcing region.

gap? If the solutions become time-dependent, what is the dominant mode of variabilty and what determines its characteristics? The approach taken here is to construct a very simple model of an ocean circulation that represents the basic elements required to address the above questions. It necessarily lacks many aspects of the real ocean circulation and forcing that motivate this study, but its simplicity provides a means to systematically vary a few controlling parameters to explore the nonlinear regime, and to construct a simple scaling to provide insight into the basic physics of this system.

\section{Model configuration}

The minimal elements required to address the questions raised in the Introduction are nonlinear zonal flows and the ability to distinguish between eastward and westward influences. The second condition can be achieved by introducing a topographic feature in the basin interior that provides a source of westward propagating characteristics and by having a source of strong zonal flows at the western boundary that provides a source of eastward propagating characteristics.

While these conditions can arise in a purely wind-driven ocean circulation in a closed basin, such flows are inherently very complex. A simpler configuration will be used here that allows for explicit control over the strength of the zonal flow without controlling the separation point from the western boundary. It also allows for strongly nonlinear flows to extend all the way to the topography located in mid-ocean. The model consists of the shallow water equations on a beta-plane in a $2500 \mathrm{~km}$ (longitude) by $2000 \mathrm{~km}$ (latitude) domain, as shown in Figure 1. There is topography that extends to the ocean surface, separated in the zonal direction by a distance $L_{x}=1300 \mathrm{~km}$. Each of these ridges is interrupted by a gap of width $\Delta=100 \mathrm{~km}$, the western gap is at latitude $y_{0}=450 \mathrm{~km}$ and the eastern gap is at latitude $y_{1}=1450 \mathrm{~km}$. The lateral boundary conditions are no-slip and no normal flow on 
all solid boundaries. The model is periodic in the zonal direction. Analysis is done on the western sub-basin, the eastern sub-basin is provided in order to close the circulation and allow for periodic boundary conditions.

There is a single moving layer of fluid of thickness $h$ with a reduced gravity of $g^{\prime}$. The equations of motion are

$$
\begin{gathered}
\vec{v}_{t}+\vec{v} \cdot \nabla \vec{v}+f \mathbf{k} \times \vec{v}=-g^{\prime} \nabla h+A_{h} \nabla^{2} \vec{v}+A_{b} \nabla^{4} \vec{v}-\gamma\left(\vec{v}-\vec{v}^{*}\right)-C_{D} \vec{v}, \\
h_{t}+\nabla \cdot(\vec{v} h)=-\gamma\left(h-h^{*}\right) .
\end{gathered}
$$

The terms on the right hand side proportional to $\gamma$ are used to force the model within the lightly shaded region in the western gap (Fig. 1). Outside of this region $\gamma=0$ and the equations are unforced. Within the shaded region, the velocity is strongly restored towards a purely zonal flow given by

$$
\vec{v}^{*}(y)=U_{m} \sin \left(\frac{\pi\left(y-y_{s}\right)}{\Delta}\right) \vec{i}
$$

where $U_{m}$ is the specified maximum inflow velocity, $y_{s}$ is the latitude of the southern edge of the gap, and the restoring constant $\gamma=1 / 600 \mathrm{~s}$. The transport per unit depth through the gap is $\Phi=(2 / \pi) U_{m} \Delta$. The layer thickness is forced towards a meridional profile that is in geostrophic balance with (3) as

$$
h^{*}(y)=H_{0}+\left(\frac{\Delta U_{m} f}{\pi g^{\prime}}\right) \cos \left(\frac{\left(y-y_{s}\right) \pi}{\Delta}\right)
$$

where $H_{0}$ is the initial resting layer thickness.

There are three forms of dissipation on the right hand side of (1). Either the Laplacian or the biharmonic terms are used, but only one is active in each calculation. When Laplacian viscosity is used, $A_{h}=200 \mathrm{~m}^{2} \mathrm{~s}^{-1}$ and $A_{b}=0$. In these cases, a Reynolds number can be defined as a measure of the nonlinearity of the flow as $R=\Phi / A_{h}$, as in Ierley and Young (1991). When biharmonic viscosity is used, $A_{h}=0$ and $A_{b}=-10^{9} \mathrm{~m}^{4} \mathrm{~s}^{-1}$. The dominant behavior of the system is not overly sensitive to decreases in viscosity, at least within the limits that can be explored at this grid spacing (discussed further below). This relatively large value of Laplacian viscosity is used in this section just to demonstrate the transition between regimes in terms of the familiar Reynolds number. Less damped calculations with biharmonic friction are used in the remainder of the paper. There is also a linear drag term that is only applied to the east of the eastern ridge, for $x>1850 \mathrm{~km}$, with $C_{D}=10^{-5} \mathrm{~s}^{-1}$. This suppresses variability in the eastern basin and allows for a smooth transition from the outflow in the northern gap to the inflow in the southern gap. This ensures that any variability in the western basin results from dynamics internal to the basin, not from remote variability propagating westward from the eastern basin through the gap at $y_{1}$. This eastern sub-domain connects the transport between the two gaps and allows for periodic boundary conditions in the model without specifying the flow through the eastern gap. This is important because, 
while the mean transport through the eastern gap is required to match that through the western gap, the potential vorticity flux through the eastern gap can not be specified and, as will be shown below, depends on the model parameters and the circulation in the western basin.

The Coriolis parameter is given by

$$
f(y)=f_{0}+\beta\left(y-y_{m}\right)
$$

where $f_{0}=10^{-4} \mathrm{~s}^{-1}, \beta=2 \times 10^{-11} \mathrm{~m}^{-1} \mathrm{~s}^{-1}$, and $y_{m}=10^{6} \mathrm{~m}$ at the central latitude of the domain. The initial layer thickness $H_{0}=1000 \mathrm{~m}$ and the reduced gravity $g^{\prime}=0.025 \mathrm{~m} \mathrm{~s}^{-2}$ (these will be varied in the next section). This gives a baroclinic deformation radius of $L_{d}=\left(g^{\prime} H_{0}\right)^{1 / 2} / f_{0}=50 \mathrm{~km}$ and a baroclinic Rossby wave speed at the mid-latitude of the basin of $5 \mathrm{~cm} \mathrm{~s}^{-1}$.

Equations (1) and (2) are solved as written on a centered difference C-grid using a third order Adams-Bashforth time stepping scheme. The horizontal grid spacing is $5 \mathrm{~km}$. The model is initialized at rest and run for a period of 100 years. Output is saved every 20 days, producing 1,800 instantaneous fields over the course of integration.

This is clearly a great simplification compared to the real ocean. While this configuration represents an extreme in that the topography consists of peninsulas, it does provide a useful limit to study the interaction of nonlinear flows with waves in the interior, taking into consideration the tendency for fluid to flow over deep gaps rather than over topographic ridges, even when the ridges are very deep (Bower and von Appen, 2008). Many realistic elements are neglected here, such as surface forcing due to winds or buoyancy fluxes, finite bottom topography ${ }^{2}$, and an active deep layer. Each of these features are likely to be important in some circumstances but, as will be shown below, even this relatively simple system exhibits complex mean and time-dependent behavior such that understanding this seemingly minimal system is warranted before considering these additional complexities.

\section{Mean fields}

The mean streamfunction and potential vorticity in the western basin for a weakly forced case with $U_{m}=0.001 \mathrm{~m} \mathrm{~s}^{-1}$ and $A_{h}=200 \mathrm{~m}^{2} \mathrm{~s}^{-1}$ is shown in Figure $2(\mathrm{R}=0.32)$. In this case the maximum zonal velocity is much less than the baroclinic Rossby wave speed and the linear solution is recovered. The pressure on the eastern ridge segments is constant, as required by no-normal flow and geostrophy. This pressure signal propagates westward at the baroclinic wave speed, leaving behind the steady solution of no meridional flow required in the unforced interior. This results in a current that extends from the inflow gap, along the western boundary to the latitude of the outflowing gap, where it turns to the east and flows through the gap in the eastern ridge. The width of the western boundary

2. A similar calculation has been done with a $500 \mathrm{~m}$ tall ridge at $x=1,800 \mathrm{~km}$ instead of topography that extends to the surface. The mean and variability of this calculation are similar to those discussed below, lending confidence that the physics in the present configuration remain relevant to the case with finite interior topography. 

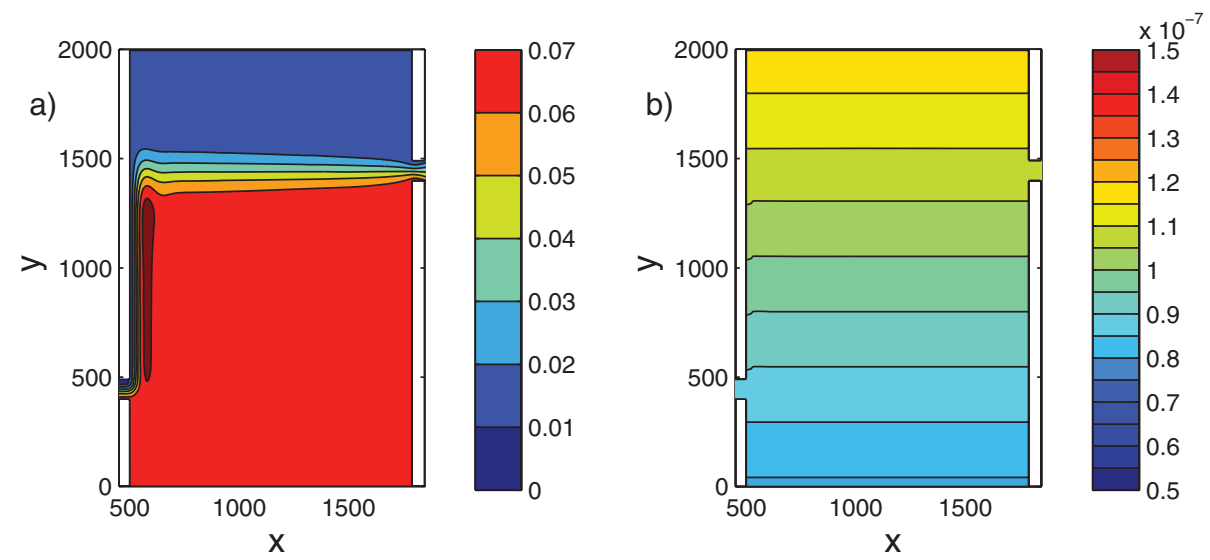

Figure 2. Mean (a) streamfunction (Sv) and (b) potential vorticity $\left(m^{-1} s^{-1}\right)$ for inflow strength $U_{m}=0.001 \mathrm{~m} \mathrm{~s}^{-1}, \mathrm{R}=0.32$.
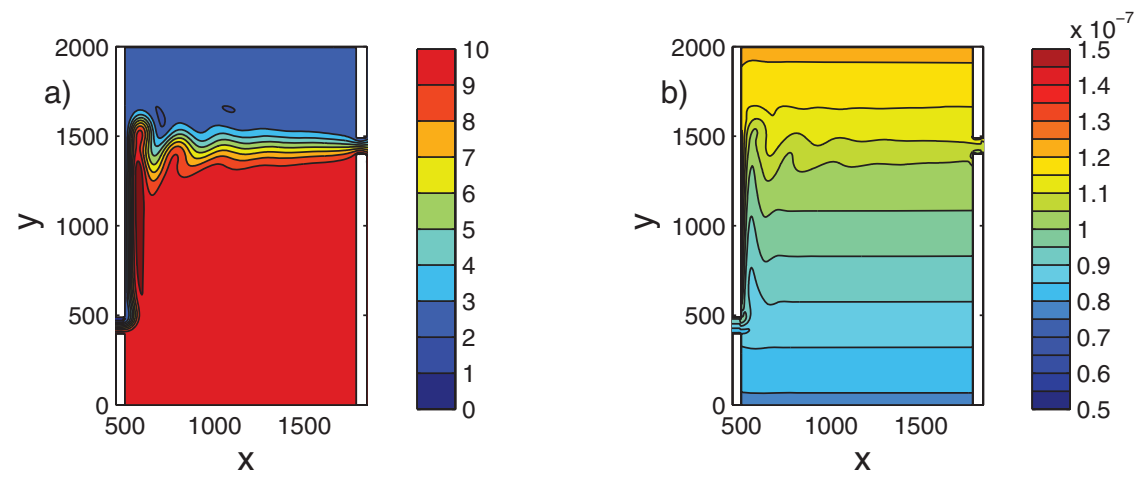

Figure 3. Mean (a) streamfunction (Sv) and (b) potential vorticity $\left(m^{-1} s^{-1}\right)$ for inflow strength $U_{m}=0.15 \mathrm{~m} \mathrm{~s}^{-1}, \mathrm{R}=47.5$.

current scales with the viscous boundary layer width, proportional to $\left(A_{h} / \beta\right)^{1 / 3} \approx 21 \mathrm{~km}$. The eastward flow is carried in a narrow jet that broadens westward from the eastern gap due to lateral viscosity. The potential vorticity is dominated by the planetary vorticity. The outflowing potential vorticity is higher than the inflowing potential vorticity because the potential vorticity of the fluid is increased as it flows northward in the western boundary layer, where the no-slip boundary condition adds positive relative vorticity to the fluid.

If the Reynolds number is increased to $47.5\left(U_{m}=0.15 \mathrm{~m} \mathrm{~s}^{-1}\right)$, the solutions remain steady but the flow develops stationary meanders near the separation point at the latitude of the outflowing gap (Fig. 3). The flow along the western boundary overshoots the outflowing latitude, retroflects in a sharp meander, and feeds into a pattern of standing meanders of 

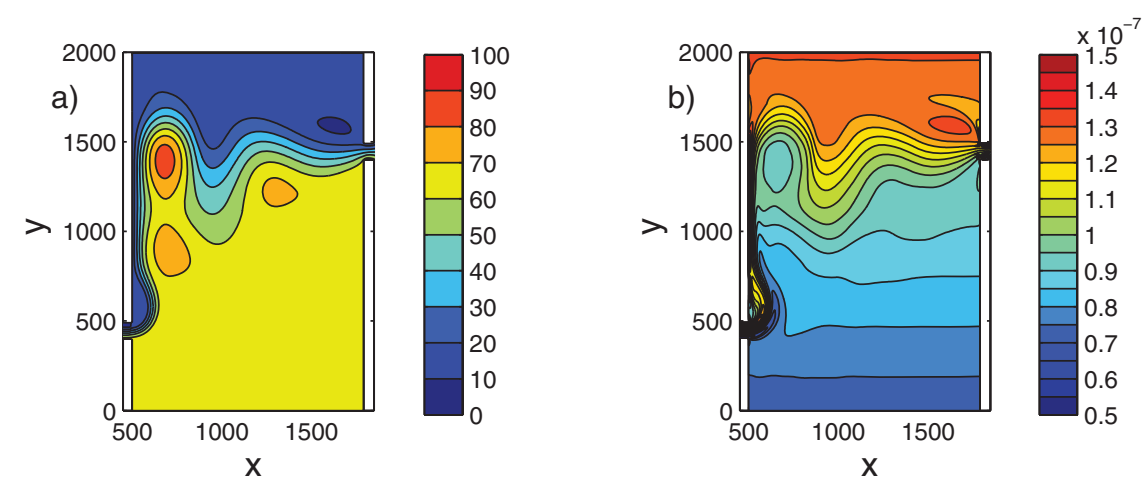

Figure 4. Mean (a) streamfunction (Sv) and (b) potential vorticity $\left(m^{-1} s^{-1}\right)$ for inflow strength $U_{m}=1 \mathrm{~m} \mathrm{~s}^{-1}, \mathrm{R}=320$.

decreasing amplitude into the basin interior. High potential vorticity is advected northward in the viscous sublayer along the western boundary and low potential vorticity is advected northwards on the offshore side of the western boundary current. This pattern is quite similar to the stationary Rossby wave solutions and meandering patterns discussed by Moore (1963) and Cessi et al. (1990). In these steady models, if the dissipation is weak enough the meandering pattern is required to modify the low potential vorticity that is carried northward in the western boundary current so that the flow exiting the boundary layer can match that in the interior, which is controlled by the planetary vorticity gradient.

Note that, even though $U_{m}$ is greater than the Rossby wave speed of $5 \mathrm{~cm} \mathrm{~s}^{-1}$, the inflow does not penetrate significantly into the basin but instead turns sharply to the north after entering through the southern gap. It is straightforward to show that there is no steady solution for a nonzonal advective pathway through the basin interior to connect the inflowing and outflowing gaps, even when the flow is nonlinear. This is a result of the twin constraints of conserving mass transport and conserving potential vorticity.

If the Reynolds number is further increased to $320\left(U_{m}=1 \mathrm{~m} \mathrm{~s}^{-1}\right)$ the flow becomes strongly nonlinear. The mean streamfunction and potential vorticity for this case are shown in Figure 4. Even with this strong inflow, the mean flow turns sharply to the north shortly after entering the basin and flows along the western boundary. The flow slightly overshoots the latitude of the eastern gap, retroflects and turns towards the east where it flows through the gap. The mean eastward flow is broadened compared to the linear solution in Figure 2 due to time-dependence and there are relatively weak mean recirculation gyres flanking the eastward flow. This pattern is similar to that found just before the onset of time-dependence, although the zonal wavelength of the meander is much larger. Similar mean flow patterns are found for calculations with the lateral viscosity reduced to $A_{h}=20 \mathrm{~m}^{2} \mathrm{~s}^{-1}(R=3200)$, although the recirculation gyres are somewhat stronger. The potential vorticity in the interior at the latitude of the jet is dominated by the layer thickness, not the planetary vorticity 


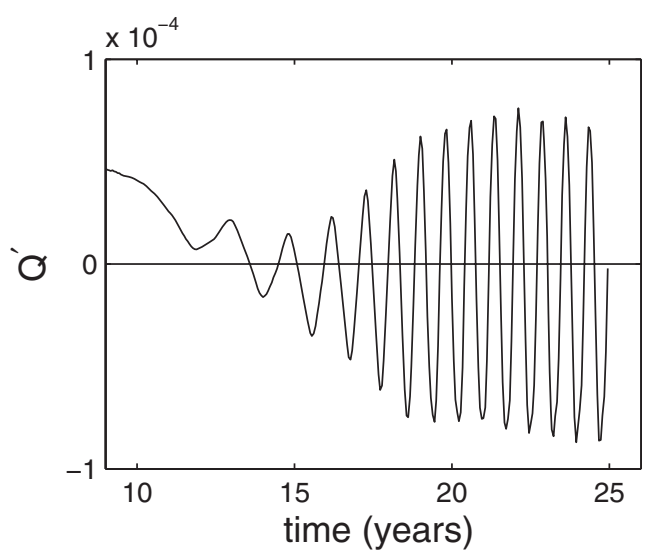

Figure 5. The basin-averaged potential vorticity anomaly for $R=50$.

(Fig. 4b). The average inflowing potential vorticity is $9.2 \times 10^{-8} \mathrm{~m}^{-1} \mathrm{~s}^{-1}$ and the average outflowing potential vorticity is $10.0 \times 10^{-8} \mathrm{~m}^{-1} \mathrm{~s}^{-1}$. Thus in the nonlinear regime the potential vorticity is modified by only about half as much as it was in the linear case and the outflowing potential vorticity can not be specified as a boundary condition to the flow. This difference compared to the linear solution arises because only part of the flow in the western boundary layer is being modified by friction in the viscous sublayer, which is narrower than the inertial boundary layer thickness that controls the width of the transport boundary layer in the nonlinear case (as in Pedlosky, 1998).

\section{Time dependence}

When the viscous flux in the western boundary layer is not sufficient to alter the potential vorticity of the inflowing fluid to match the potential vorticity in the interior the linear solution is no longer valid. In the steady models of Moore (1963) and Cessi et al. (1990), and the present model for moderate Reynolds numbers, the flow develops standing meanders that effectively increase the ability of dissipation to alter the potential vorticity of the fluid. If the Reynolds number is increased slightly beyond that in Figure 3 to $R=50$, the solution becomes time-dependent. The mean streamfunction looks much like that for $R=47.5$ but now the meanders begin to grow and propagate to the west. A time series of the basinaveraged potential vorticity anomaly, normalized by $f_{0} / H_{0}$, shows that the oscillations develop after about 15 years of integration (Fig. 5). The anomaly changes sign as the perturbations interact with the western boundary, introducing cyclonic and anticyclonic vorticity into the fluid. The oscillation grows in amplitude and eventually equilibrates at somewhat less than $10^{-4}$. The period of the oscillation is about 270 days. Ierley and Young (1991) found that instabilities of a western boundary current can develop for Reynolds numbers between about 22 and 50, depending on details of the velocity profile of the 

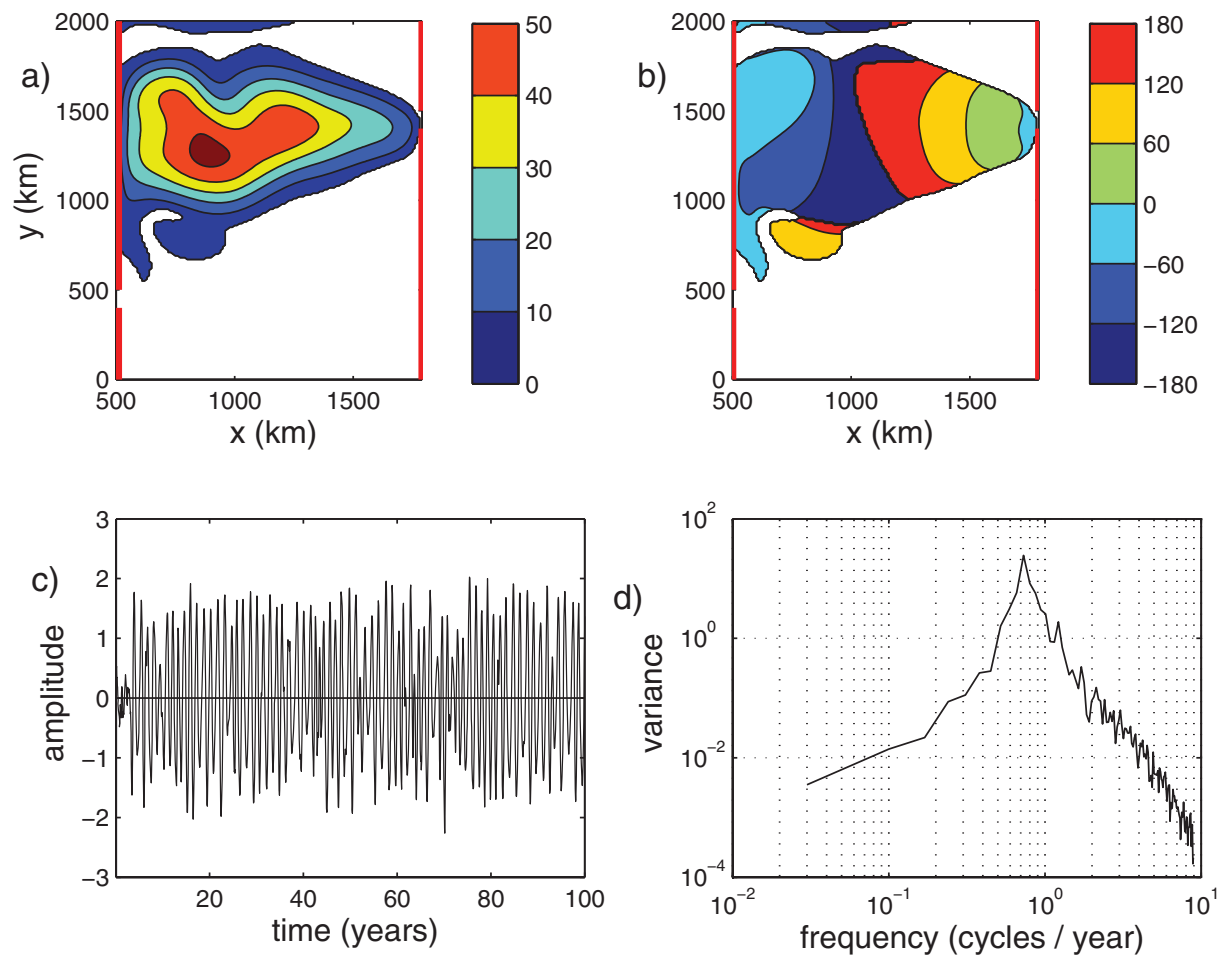

Figure 6. Leading mode for complex Empirical Orthogonal Function analysis. (a) amplitude pattern (Sv); (b) phase (degrees); (c) time series of amplitude; (d) temporal variance of the amplitude. Red lines in (a) and (b) indicate the locations of the solid boundaries.

boundary current. The onset of time-dependence in the present model is consistent with their stability analysis. Understanding the amplitude and period of these oscillations is the focus of this section.

The characteristics of the variability are demonstrated by the case with $U_{m}=1 \mathrm{~m} \mathrm{~s}^{-1}$ and biharmonic viscosity $\left(A_{h}=0, A_{b}=-10^{9} \mathrm{~m}^{4} \mathrm{~s}^{-1}\right)$. This calculation is quite similar to the previous case with Laplacian viscosity in Figure 4 but the variability is less damped. The sensitivity experiments below all use this value of biharmonic viscosity. The pattern of variability is indicated by taking the Complex Empirical Orthogonal Function (CEOF) of the streamfunction field (Barnett, 1983). This provides a two-dimensional pattern of variability, a two-dimensional phase relationship, and a time-dependent amplitude for each independent mode of variability.

The leading mode, which explains $40 \%$ of the variability in the model, is shown in Figure 6. Regions where the amplitude is less than $5 \%$ of the maximum are left blank. The maximum variability is found near the latitude of the eastern gap, broadly distributed 

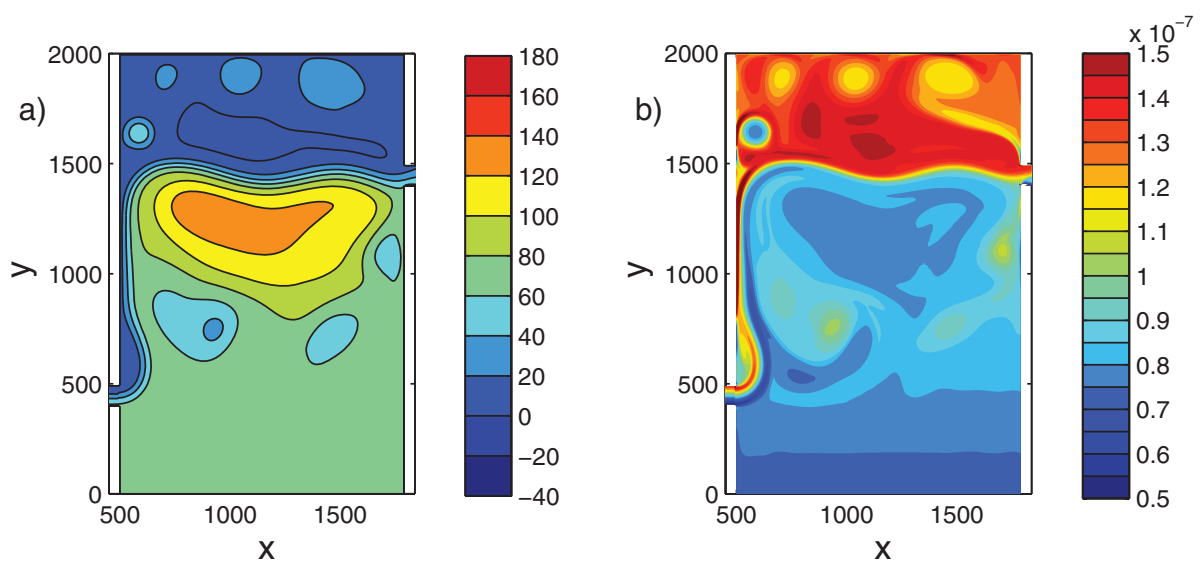

Figure 7. Instantaneous (a) streamfunction (Sv) and (b) potential vorticity $\left(m^{-1} s^{-1}\right)$ at Year 28.

across the basin but a maximum about $400 \mathrm{~km}$ off the western boundary. The magnitude of this fluctuation is $O(50 \mathrm{~Sv})$, or the same strength as the mean flow. This indicates that the circulation pattern is highly time dependent and the mean flow changes significantly on the time scale of this mode. The phase relationship (Fig. 6b) shows a propagation from east to west. The phase starts near zero at the eastern boundary and increases westward, nearly independent of latitude. There is a small signal coming from the south near $x=800 \mathrm{~km}$, but its amplitude is quite weak. The wavelength of this mode is calculated by taking twice the distance from the phase equals zero longitude near the outflow to the phase equals $180^{\circ}$ near $x=1500 \mathrm{~km}$. Although there is only weak dependence on latitude, this calculation was done at the latitude of the outflowing gap, $y_{1}=1,450 \mathrm{~km}$. The resulting wavelength is $1,260 \mathrm{~km}$. This compares reasonably well with the scaling wavelength from Moore (1963) and Cessi et al. (1990) of $L_{R W}=2 \pi\left(U_{m} / \beta\right)^{1 / 2}=1,400 \mathrm{~km}$.

This pattern of variability is dominated by a single frequency, as shown by the time series of the mode amplitude in Figure 6c. The spectra of this time series has a well defined peak at 0.73 cycles per year (Fig. 6d), or a period of 1.4 years. The phase speed of the westward propagating signal is calculated as $c=1.26 \times 10^{6} \mathrm{~m} / 1.4$ years $=3 \mathrm{~cm} \mathrm{~s}^{-1}$. This is similar to, but lower than, the baroclinic Rossby wave speed of $4.3 \mathrm{~cm} \mathrm{~s}^{-1}$ at the outflow latitude of $y=1450 \mathrm{~km}$. The difference is likely due to the finite meridional wavelength of the perturbations, which will slow Rossby waves compared to the long wave limit.

The pattern of the variability is indicated by the streamfunction and potential vorticity at three phases of the oscillation. On day 10,080 (Year 28) the CEOF has an amplitude of approximately -1 . In this phase, the streamfunction flows along the western boundary to latitude $y_{1}$, turns sharply to the east, and flows out the eastern gap (Fig. 7a). There is a large anticyclonic recirculation in the basin interior. The potential vorticity is dominated by a narrow front separating well mixed low values to the south and high values to the north 

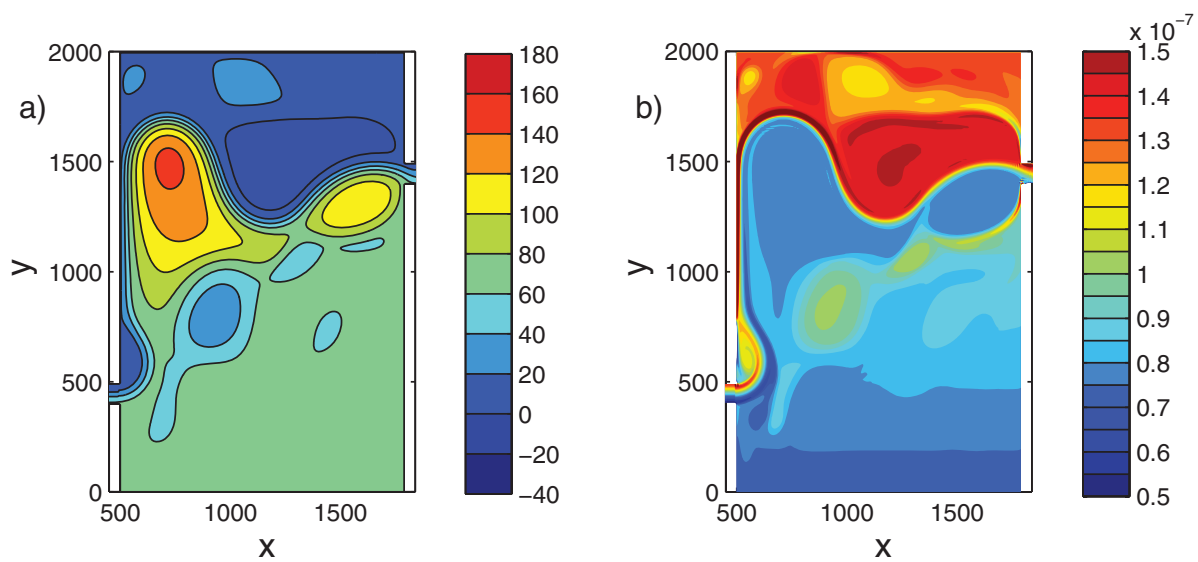

Figure 8. Instantaneous (a) streamfunction (Sv) and (b) potential vorticity $\left(m^{-1} s^{-1}\right)$ at Year 28.4.

(Fig. 7b). There is a remnant of an anticyclonic eddy near the western boundary at about $1,700 \mathrm{~km}$ latitude. 150 days later (Year 28.4), the flow along the western boundary has penetrated 200 to $300 \mathrm{~km}$ northward of latitude $y_{1}$ and the anticyclonic recirculation has shifted westward (Fig. 8a). The flow along the western boundary is producing a thin layer of high potential vorticity which is then being advected into the basin interior in the separated jet (Fig. 8b). This is feeding cyclonic relative vorticity into the cyclonic meander that is developing in the middle of the basin. This cyclonic meander continues to develop and propagate westward so that 210 days later (Year 29, 360 days after the time in Figure 7) the northward meander along the western boundary has pinched off and formed an anticyclonic eddy to the north (Fig. 9a). The cyclonic recirculation has now propagated all the way to the western boundary and the transport from the inflowing gap to the outflowing gap takes a more interior pathway. The cyclonic recirculation is producing low potential vorticity along the western boundary, which is advected into the basin interior, reducing the cyclonic vorticity in the recirculation. The flow returns to the state shown in Figure 7 as the anticyclonic eddy and cyclonic recirculation are dissipated and the inflow returns to the western boundary and penetrates northward.

The basin-averaged potential vorticity varies with the phase of the oscillation, as shown in Figure 10. The black dots mark the times of the streamfunction and potential vorticity shown in Figures 7, 8, and 9. The basin-averaged potential vorticity is low when the anticyclone dominates the circulation, the flow path is relatively straight, and the CEOF is negative. Approximately $1 / 4$ of a cycle later the average potential vorticity anomaly is nearly zero but increasing, and the CEOF has transitioned from negative to positive. In this phase there is a large anticyclonic/cyclonic pair of meanders (Fig. 8). Interaction with the western boundary is producing positive relative vorticity, which is then being advected into the basin interior by the separated front. After shedding an anticyclonic eddy to the north, the flow takes the 

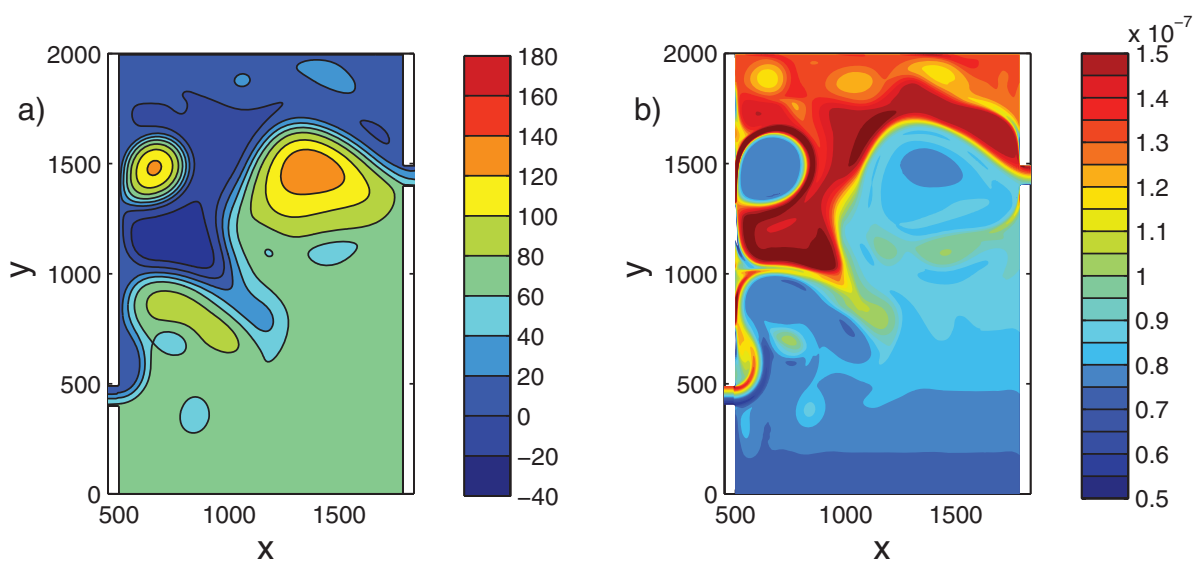

Figure 9. Instantaneous (a) streamfunction (Sv) and (b) potential vorticity $\left(m^{-1} s^{-1}\right)$ at Year 29.

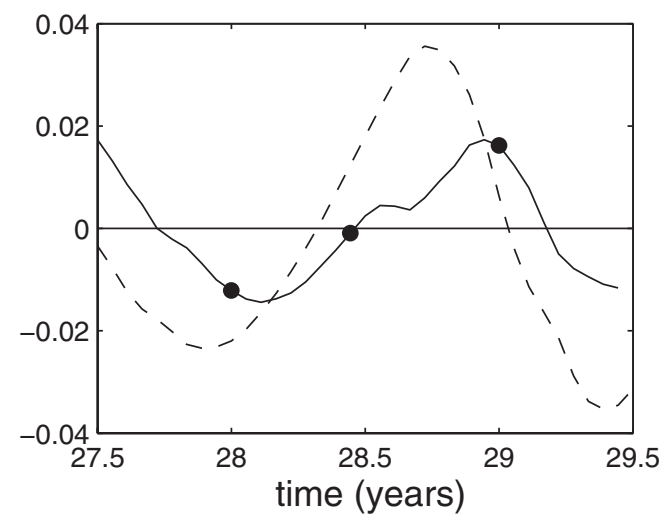

Figure 10. Basin-averaged potential vorticity anomaly (normalized by $f_{0} / H_{0}$, solid line) and amplitude of the CEOF (divided by 50, dashed line) for a two year period. Dots are placed at the times shown in Figures 7 to 9 (Year 28, 28.4, and 29).

interior path, the anticyclone is dissipated, and the average potential vorticity anomaly is positive.

The full time series of the basin-averaged potential vorticity anomaly is shown in Figure 11a. The variability is dominated by the oscillation detailed in Figures 7 to 10 . This time series has a spectral peak at 0.73 cycles/year, the same as was found for the leading mode of the CEOF analysis (Fig. 11b). The amplitude of the peak is $6 \times 10^{-18} \mathrm{~m}^{-2} \mathrm{~s}^{-2}$. The typical potential vorticity anomaly of the oscillation averaged over the active model domain is the square root of this variance, or $2.5 \times 10^{-9} \mathrm{~m}^{-1} \mathrm{~s}^{-1}$. The potential vorticity 
a)
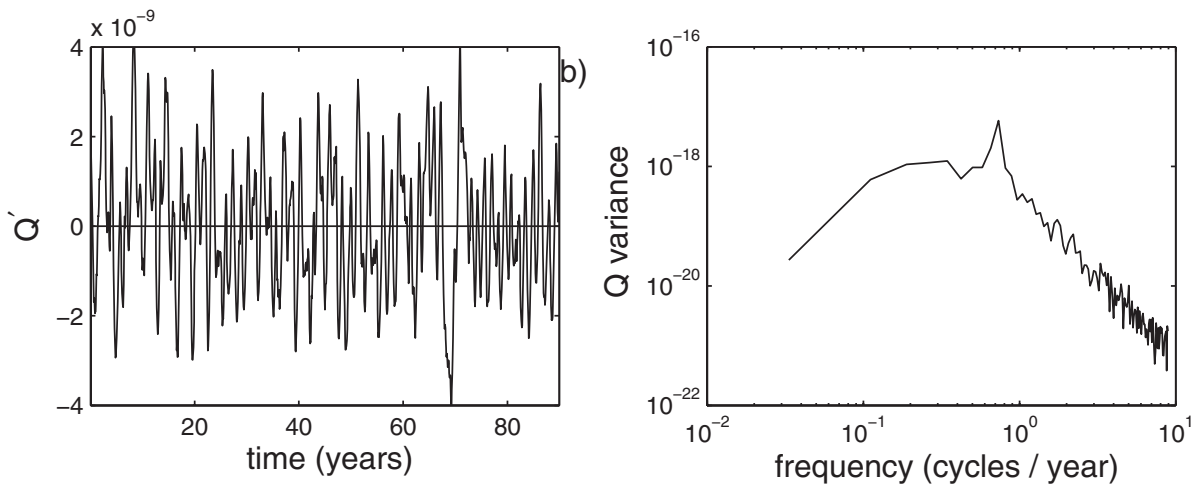

Figure 11. (a) Time series of the basin-averaged potential vorticity. (b) Spectral distribution of the temporal variance of the basin-averaged potential vorticity.

at the mid-latitude of the basin for the resting layer thickness of $1,000 \mathrm{~m}$ is $10^{-7} \mathrm{~m}^{-1} \mathrm{~s}^{-1}$, so the basin-averaged anomaly is typically $2.5 \%$ of the mean.

The oscillation is described as a series of cyclonic and anticyclonic meanders that develop in the basin interior and propagate westward. Their interaction with the no-slip western boundary layer produces excess vorticity of the opposite sign, which is advected into the basin interior, leading to the development of the next phase of the oscillation. Similar oscillations are found over a wide range of parameter space (presented below). This connection between the oscillation and the basin averaged potential vorticity suggests that the underlying mechanism relies on the modification of potential vorticity due to the boundary and is thus fundamentally different from instabilities arising in the interior of the basin or the eddyshedding process discussed by Nof and coworkers (e.g. Zharkov and Nof 2008). A simple expression will now be developed to predict the amplitude and period of the oscillation and its dependence on the model parameters.

The potential vorticity is modified in a viscous sublayer that is narrower than the width of the western boundary current. In this sublayer, the balance in the potential vorticity equation is between meridional advection and lateral dissipation (as in Pedlosky, 1998). For a general form of dissipation proportional to the $n^{\text {th }}$ derivative of the streamfunction, this balance is written as

$$
U \zeta_{y}=v \zeta_{n}
$$

where $U$ is the velocity scale of the western boundary current, $\nu$ is the viscosity coefficient, $\zeta_{y}$ is the meridional gradient in the relative vorticity, and $\zeta_{n}$ is the $n^{\text {th }}$ zonal derivative of the relative vorticity. The width of the viscous sublayer is then calculated as

$$
\delta_{*}=\left(\frac{\nu L}{U}\right)^{1 / n}
$$


where zonal derivatives are proportional to $\delta_{*}^{-1}$ and $L$ is the meridional scale of variations in the boundary current. Consistent with boundary layer scaling, it is assumed that $\delta_{*} \ll L$.

The velocity scale of the western boundary current $U$ is derived from the width of the western boundary current, taken to be the inertial boundary layer width $\delta_{I}=(U / \beta)^{1 / 2}$. A second constraint arises if it is required that the transport through the southern gap is the same as that carried in the western boundary current, $\Phi=\delta_{I} U$. Combining these two conditions, the velocity scale in the boundary layer is then

$$
U=\Phi^{2 / 3} \beta^{1 / 3}
$$

The change in potential vorticity of the fluid being advected in the viscous sublayer can be calculated as the product of the rate of vorticity production in the viscous sublayer $\left(\nu \zeta_{n} / h\right)$ and the amount of time the fluid is in the boundary current $(L / U)$.

$$
\Delta q=\frac{\nu \zeta_{n}}{h} \frac{L}{U}=\frac{\nu L}{h \delta_{*}^{n+1}}
$$

If the viscosity is second order $(n=2)$ and the width of the viscous sublayer is the same as the Munk layer $\left(\delta_{*}=(\nu / \beta)^{1 / 3}\right)$, then the change in potential vorticity in the boundary current is $\beta L / h$, the same as the change in the planetary vorticity over a meridional distance $L$. This is the balance for the linear solution. However, if the viscous sublayer is narrower than $(\nu / \beta)^{1 / 3}$ the vorticity produced in the sublayer will be in excess of the change required to smoothly match the interior potential vorticity. The steady models of Moore (1963) and Cessi et al. (1990) balance the potential vorticity budget by increasing the length over which viscosity can act through the development of standing meanders, within which this excess positive relative vorticity is mixed with low potential vorticity advected within the boundary current that is outside the viscous sublayer.

The flux of potential vorticity from the viscous sublayer into the basin interior is given by

$$
\Delta \dot{Q}=\delta_{*} U h \Delta q=\frac{\nu U L}{\delta_{*}^{n}}=U^{2} .
$$

The vorticity production in the viscous sublayer is simply proportional to the velocity scale of the boundary current squared. It is independent of the specific form of friction, the meridional offset between the gaps $L$, and the viscosity coefficient.

The amplitude of the oscillation is given by the integral of this flux of anomalous vorticity over the time period for which the flow is in the phase of the oscillation with northward flow along the western boundary. We will take this time scale to be the period of the oscillation, which is proportional to the standing Rossby wave zonal wavelength $L_{R W}=2 \pi(U / \beta)^{1 / 2}$ divided by the phase speed of baroclinic Rossby waves.

$$
T=\frac{L_{R W}}{c}=2 \pi\left(\frac{U}{\beta}\right)^{1 / 2} \frac{f_{0}^{2}}{g^{\prime} H_{0} \beta}
$$


This is a natural time scale in the system to use for the scaling analysis. The phase speed that sets the period of the oscillation is the baroclinic Rossby wave speed because the oscillation is marked by the westward propagation of potential vorticity anomalies from the interior of the basin to the western boundary. These anomalies are advected eastward in the narrow jet but accumulate in the larger recirculations that propagate westward, uninfluenced by the narrow jet. The length scale to use is less clear. The standing Rossby wavelength is used here even though the meanders in the system are not stationary. The baroclinic deformation radius, the inertial length scale, and the basin width were also tried in the scaling but the results did not fit the model data very well.

Before testing (11), the wavelength of the westward propagating meanders was calculated from the leading CEOF, as in Fig. 6, for a series of 32 model calculations in which the basic model parameters have been varied. The inflow velocity has been varied between $0.2 \mathrm{~m} \mathrm{~s}^{-1}$ and $2 \mathrm{~m} \mathrm{~s}^{-1}$, the Coriolis parameter between $0.5 \times 10^{-4} \mathrm{~s}^{-1}$ and $1.5 \times 10^{-4} \mathrm{~s}^{-1}$, the reduced gravity between $0.0125 \mathrm{~m} \mathrm{~s}^{-2}$ and $0.1 \mathrm{~m} \mathrm{~s}^{-2}$, the variation in planetary vorticity between $1 \times 10^{-11} \mathrm{~m}^{-1} \mathrm{~s}^{-1}$ and $3 \times 10^{-11} \mathrm{~m}^{-1} \mathrm{~s}^{-1}$, the gap offset has been set to 1,000 $\mathrm{km}$ (standard), $500 \mathrm{~km}$, and $0 \mathrm{~km}$, and the layer thickness between $750 \mathrm{~m}$ and 1,500 m. Oscillations very similar to that detailed above are found in each of these calculations.

The diagnosed wavelength of the westward propagating meanders is compared to $L_{R W}$, where $U$ is determined by (8), in Figure 12a. The central case discussed above is indicated by the solid circle, all other calculations are open circles. The scaling reproduces the parameter dependence reasonably well, the slope of the solid line is 0.8 . For the case at the extreme right, (model wavelength 2,400 km), the meridional offset between the gaps is only $500 \mathrm{~km}$ and the westward propagating meander pattern is the second leading mode of variability, not the leading mode as it is for all of the other calculations. The leading mode in this case is a north-south shift of the jet path. While it is not clear why this would change the wavelength of the westward propagating meanders, it is the only clear distinction between this case and the others for which the scaling appears to work reasonably well.

The spectral energy of the basin-averaged potential vorticity has been calculated for each case and a distinct spectral peak is found. The period predicted by (11), with $U$ from (8), is compared to that diagnosed from the spectral analysis in Figure 12b. This variation in parameters produces periods ranging from just under 100 days to over 3,000 days, providing a wide range with which to test the scaling. While the period resulting from the scaling theory is consistently shorter than is found in the model, the parameter dependence is quite well represented by the scaling.

The amplitude of the oscillation is measured by the basin averaged potential vorticity anomaly. The non-dimensional potential vorticity anomaly is the production rate times the oscillation period divided by the basin volume and normalized by the background potential vorticity of $f_{0} / H_{0}$.

$$
Q^{\prime}=\Delta \dot{Q} \frac{T}{L_{x} L_{y} H_{0}} \frac{H_{0}}{f_{0}}=\frac{U^{2}}{g^{\prime} H_{0}} \frac{f_{0}}{\beta L_{y}} \frac{L_{R W}}{L_{x}}=\frac{F^{2} \lambda}{\beta^{*}}
$$


a)

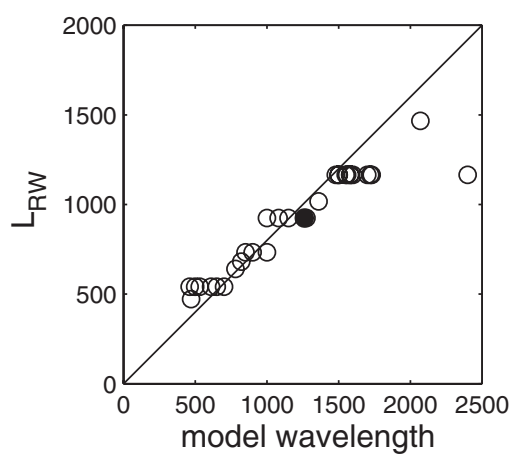

c)

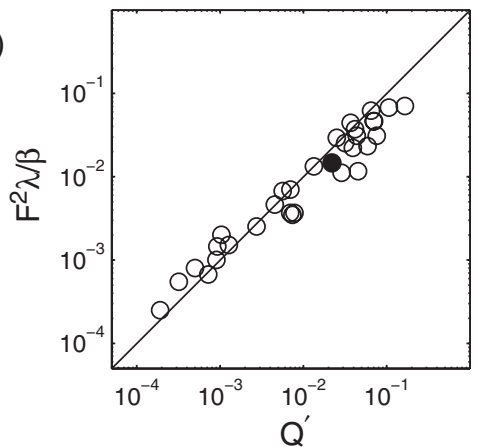

b)

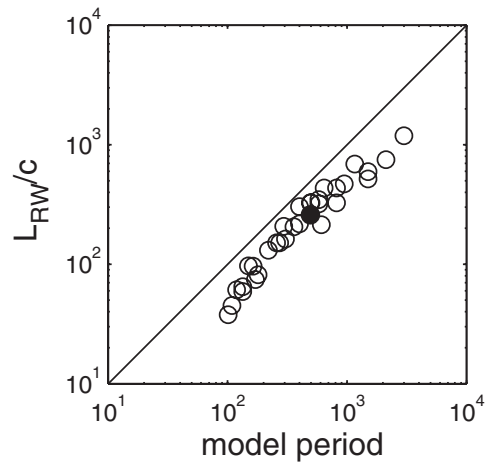

Figure 12. Comparison between (a) wavelength ( $\mathrm{km}$ ), (b) period (days), and (c) the amplitude of the potential vorticity variance produced by the numerical model and that predicted by the scaling theory (11) and (12) for a wide range of model parameters. The solid circle is the case discussed in detail in Figures 7 to 11.

where $F=U /\left(g^{\prime} H_{0}\right)^{1 / 2}$ is a Froude number, $\lambda=L_{R W} / L_{x}$ is the ratio of the standing Rossby wavelength to the zonal dimension of the basin, and $\beta^{*}=\beta L_{y} / f_{0}$ is the normalized change in planetary vorticity over the meridional extent of the domain. It is somewhat surprising that the amplitude of the oscillation does not depend on the meridional offset between the inflowing and outflowing gaps or the friction coefficient. The scaling (12), with (8), predicts the amplitude of the potential vorticity anomaly for the model configuration described above to be 0.015 . This is to be compared with the square root of the spectral peak in Fig. 11 b, normalized by $f_{0} / H_{0}$, which is 0.022 . The comparison is reasonable, but a more complete test of (12) is found by comparison for all of the model calculations in Figure 12c. The scaling theory reproduces the amplitude of the potential vorticity anomalies found in the model reasonably well over three orders of magnitude.

The level of viscosity used $\left(A_{b}=-10^{9} \mathrm{~m}^{4} \mathrm{~s}^{-1}\right)$ was chosen to provide numerically stable results with smooth potential vorticity fields for all model runs. Calculations with 

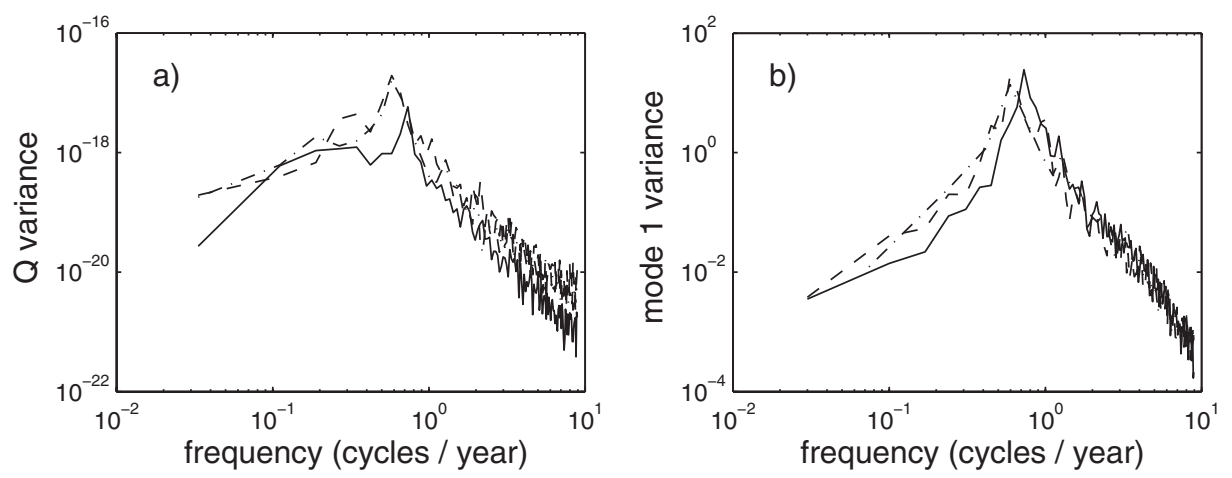

Figure 13. Spectral energy for: (a) basin-averaged potential vorticity and (b) CEOF Mode 1 amplitude. Solid line: $A_{b}=-10^{9} \mathrm{~m}^{4} \mathrm{~s}^{-1}$; dashed line: $A_{b}=-10^{8} \mathrm{~m}^{4} \mathrm{~s}^{-1}$; dot-dashed line: $A_{h}=20 \mathrm{~m}^{2} \mathrm{~s}^{-1}$

$U=1 \mathrm{~m} \mathrm{~s}^{-1}$ were able to be run with smaller values of viscosity, $A_{b}=-10^{8} \mathrm{~m}^{4} \mathrm{~s}^{-1}$ for biharmonic viscosity or $A_{h}=20 \mathrm{~m}^{2} \mathrm{~s}^{-1}(R=3,200)$ for Laplacian viscosity ${ }^{3}$. The peak spectral energy for the basin-integrated potential vorticity is about a factor of two higher, and at slightly lower frequency, for these cases than it is for the standard viscosity (Fig. 13a). There is also elevated energy at higher and lower frequencies, indicating a more variable and turbulent flow. However, the variability is dominated by the same oscillatory mode discussed above, with the leading CEOF explaining $45 \%$ of the streamfunction variance in each of these calculations. The spectral energy of the leading CEOF is quite similar to that for the standard calculation, in terms of both the amplitude and frequency of the peak (Fig. 13b), so the basic mechanism discussed above appears to be robust to modest reductions in viscosity.

There is one case that results in both low frequency variability of the basin-scale flow and the high frequency variability characterized by the above oscillation. For a strongly forced run $\left(U_{m}=2 \mathrm{~m} \mathrm{~s}^{-1}\right)$ and small meridional offset of the inflowing and outflowing gaps $\left(y_{1}-y_{0}=500 \mathrm{~km}\right)$, the circulation fluctuates between two different states. This is reminiscent of the multiple states found in purely wind-driven circulations (as in McCalpin and Haidvogel, 1996) but the mechanism here appears to be different and was only found for this particular configuration. The two leading complex EOFs explain 32\% and 30\% of the variance, so they are both important in describing the variability. The leading CEOF is fundamentally different from the westward propagating mode found previously (Fig. 14a). The amplitude now has three local maxima. The largest variance is in the middle of the basin near the inflowing gap latitude of $850 \mathrm{~km}$. There are two smaller signals near the outflowing gap latitude of $1,350 \mathrm{~km}$, shifted both east and west of the mid-longitude of the basin. The phase now shows meridional propagation from south to north, with a phase lag of about

3. These reduced values were not able to be used for the strongest forced cases reported above with $U=2 \mathrm{~m} \mathrm{~s}^{-1}$. 

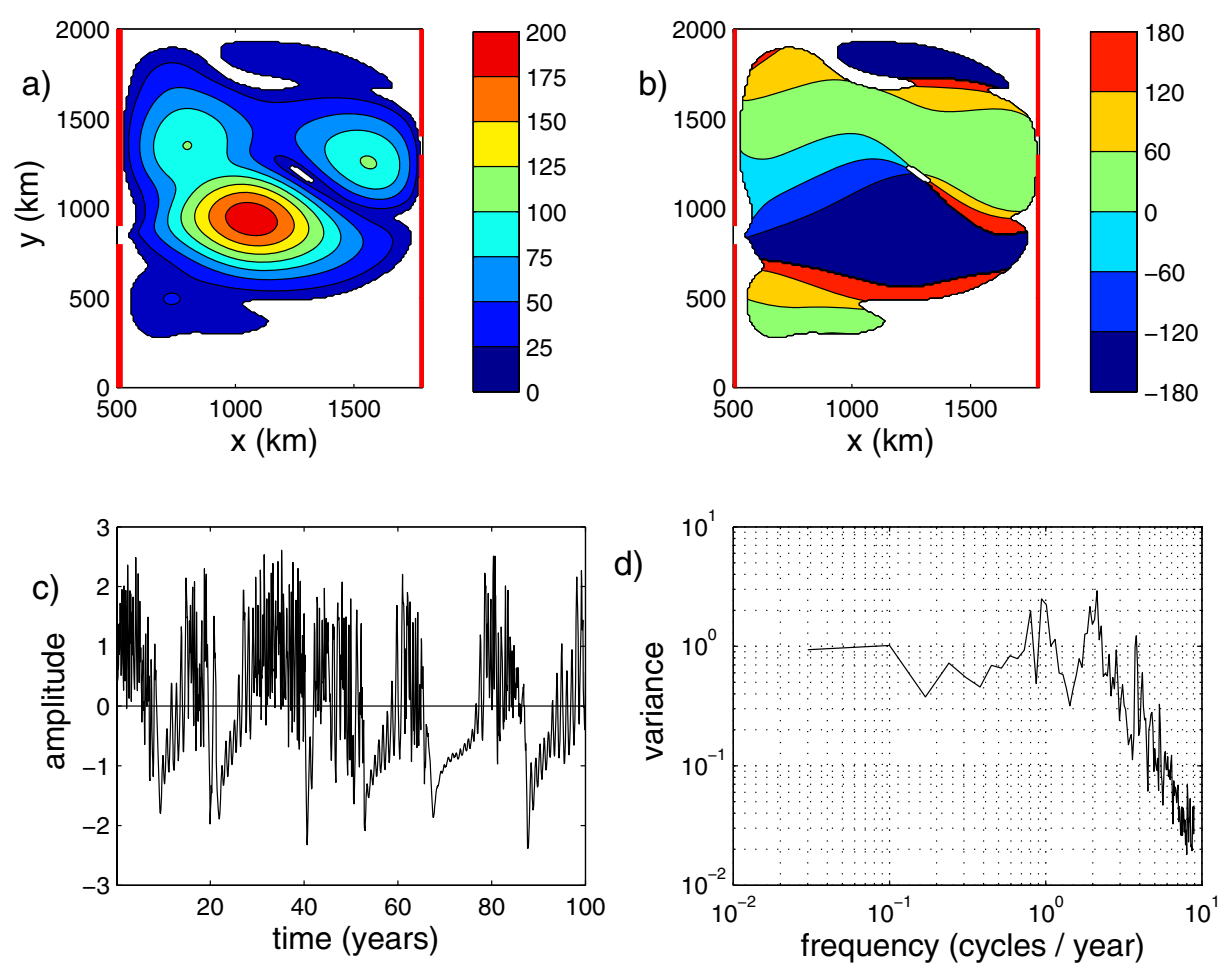

Figure 14. Leading mode for complex Empirical Orthogonal Function analysis for the case with $y_{1}-y_{0}=500 \mathrm{~km}, g^{\prime}=0.1 \mathrm{~m} \mathrm{~s}^{-2}$, and $U_{m}=2 \mathrm{~m} \mathrm{~s}^{-1}$. (a) amplitude pattern; (b) phase; (c) time series of amplitude; (d) temporal variance of the amplitude. Red lines in (a) and (b) indicate the locations of the solid boundaries.

$180^{\circ}$ across the mid-latitude of the basin (Fig. 14b). This indicates that when the southern recirculation is stronger (more anticyclonic) the northern recirculation is also stronger (more cyclonic). Thus this mode represents a double gyre pattern centered on the jet as it crosses the basin. When this mode amplitude is negative, the negative phase indicates stronger anticyclonic and cyclonic recirculations on the southern and northern sides of the front. The amplitude exhibits a saw-tooth pattern where there is a rapid transition from positive to negative values, and then a slow increase in time (Fig. 14c). Once the mode amplitude becomes positive it begins to fluctuate rapidly until it jumps back into the large, negative state. The circulation stays in these relatively low variability states for $O$ (5-10 years).

The second mode of variabilty is similar to the westward propagating mode discussed previously (Fig. 15). There is a single maximum at the outflowing gap latitude with westward phase propagation. The westward phase speed is slower than was found for the weaker inflow strength of $U_{m}=1 \mathrm{~m} \mathrm{~s}^{-1}$, giving rise to the very long wavelength of the westward propagating mode shown in Figure 12a. The mode amplitude shows rapid fluctuations with 

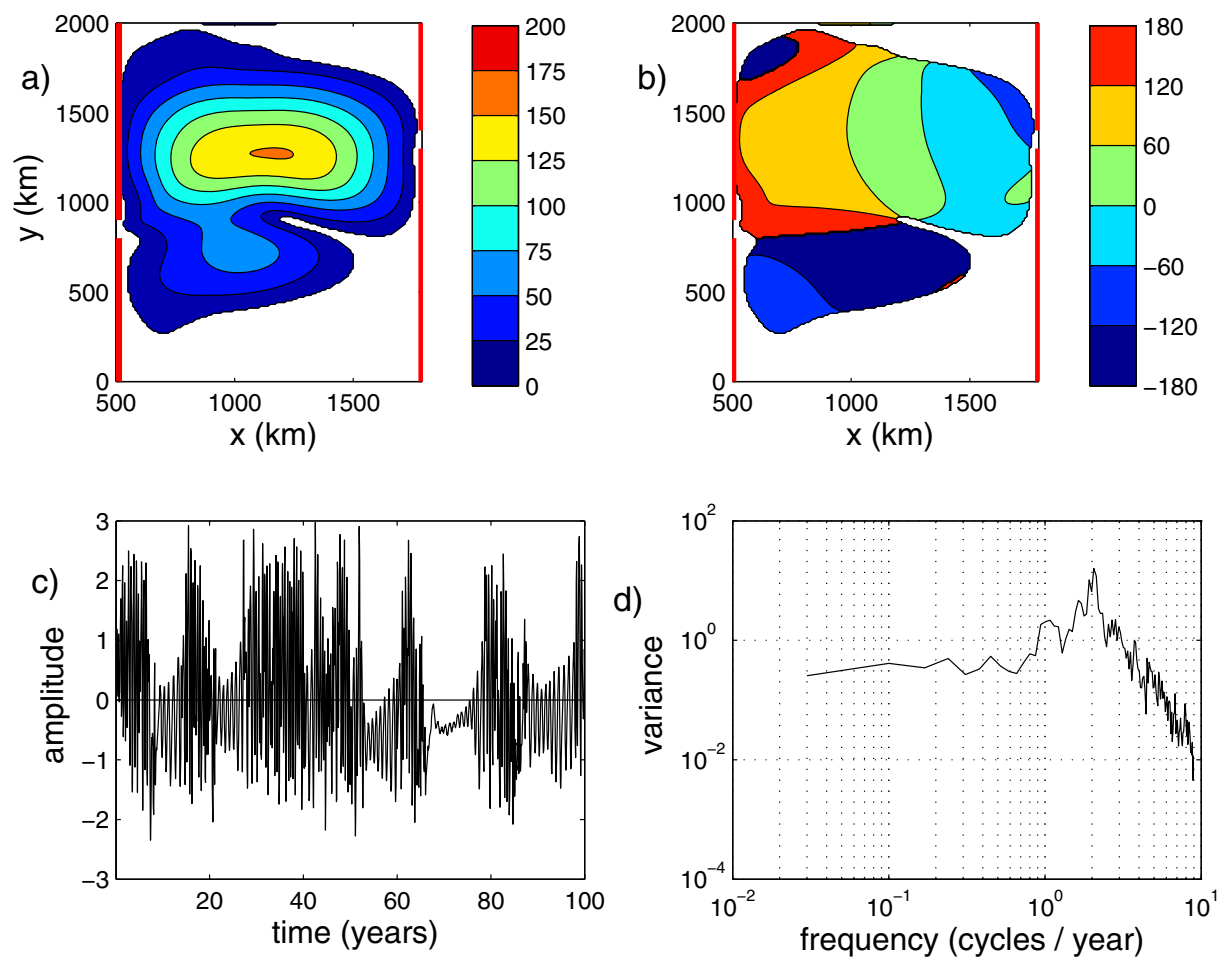

Figure 15. Second leading mode for complex Empirical Orthogonal Function analysis for the case with $y_{1}-y_{0}=500 \mathrm{~km}, g^{\prime}=0.1 \mathrm{~m} \mathrm{~s}^{-2}$, and $U_{m}=2 \mathrm{~m} \mathrm{~s}^{-1}$. (a) amplitude pattern; (b) phase; (c) time series of amplitude; (d) temporal variance of the amplitude. Red lines in (a) and (b) indicate the locations of the solid boundaries.

frequency 2.1 cycles per year, very close to the 2.0 frequency found for the same inflow strength and a gap offset of $1,000 \mathrm{~km}$. Comparison with the Mode 1 amplitude shows that Mode 2 is more active when Mode 1 is positive. The periods when the recirculation gyres are strong (negative Mode 1) are also periods of little westward wave propagation. In this state, the flow is able to cross the basin in a nearly zonal jet and maintain a northward flow near the eastern boundary to reach the outflowing gap. This northward flow is prohibited from propagating westward, as would be expected for an unforced interior, because of the cyclonic recirculation gyre to the north of the front. The streamfunction reconstructed from the mean streamfunction minus one times the Mode 1 streamfunction shows two strong recirculation gyres flanking a jet that extends all the way to the eastern boundary (Fig. 16a). In this state there is little interaction between the jet and the western boundary. When Mode 1 is plus one, the inflow turns to the north along the western boundary, as found for the large gap offsets discussed above, and supports the oscillatory mechanism (Fig. 16b). 

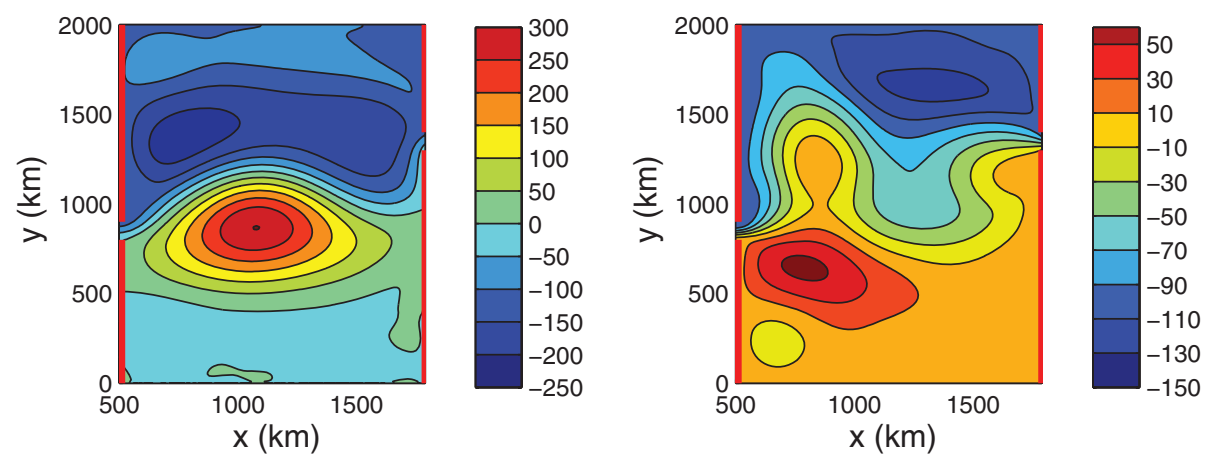

Figure 16. Reconstructed streamfunction for the case with $y_{1}-y_{0}=500 \mathrm{~km}, g^{\prime}=0.1 \mathrm{~m} \mathrm{~s}^{-2}$, and $U_{m}=2 \mathrm{~m} \mathrm{~s}^{-1}$. (a) mean minus one standard deviation of the leading mode; (b) mean plus one standard deviation of the leading mode. Red lines indicate the locations of the solid boundaries.

\section{Summary}

The influences of nonlinearity and wave propagation in setting the mean and timedependent flow between a western boundary and topography in the basin interior have been explored using a very idealized shallow water model and scaling theory. The mean path of the current follows the western boundary from the inflow latitude to the latitude at which it can exit the domain, as expected for linear flows, even for cases in which the velocity far exceeds the baroclinic wave speed. This is understood by the lack of steady nonzonal solutions in an unforced basin interior. For weakly nonlinear flows the circulation develops steady, stationary meanders just to the east of the separation point, consistent with previous studies (Moore 1963; Cessi et al., 1990). The western boundary current becomes unstable for moderate Reynolds numbers, yielding time-dependent solutions. The primary mode of variability is characterized by westward propagating meanders with maximum amplitude in the western part of the basin and eddy shedding along the western boundary. The mechanism of variability is related to vorticity input through the narrow viscous sublayer at the western boundary. This vorticity is then advected into the basin interior in the separated jet. As it is carried eastwards, it is spread meridionally in recirculation gyres adjacent to the jet. Once outside the strong eastward advection in the core of the jet the potential vorticity anomalies propagate westward to, eventually, interact with the western boundary and produce anomalous vorticity of the opposite sign. The wavelength of this meander pattern scales with the inertial boundary layer length scale $2 \pi(U / \beta)^{1 / 2}$. The period of the oscillation is determined by this wavelength and the baroclinic wave speed. The amplitude of the oscillation is predicted by a simple scaling that takes account of the vorticity input along the western boundary over the period of the oscillation. The wavelength, period, and amplitude of the oscillation compare well with a series of numerical model calculations over a wide range of parameter space. 
A very simple model was used here in order to identify and isolate the most basic elements of the problem: nonlinear advection, wave propagation, and zonal asymmetry. However, the examples in the introduction that were used to motivate this problem exist in more complicated settings and may be influenced by additional processes not considered here. Western boundary currents that separate at mid-latitudes are embedded within larger-scale wind-driven gyres. Bottom topography along the western boundary may alter the strength and parameter dependence of vorticity production within the viscous sublayer. Perhaps the biggest limitation of this model is the neglect of baroclinic instability. A necessary condition for baroclinic instability is that the potential vorticity gradient change sign within the domain. For a flat bottom, two layer fluid this condition is met when the eastward velocity of a separated jet exceeds the westward phase speed of long baroclinic Rossby waves, which is the region of parameter space in which the present oscillations arise. The degree of simplification taken here makes it relatively easy to identify and understand the mean and dominant mode of variability in the nonlinear regime but it remains to be seen how these additional physical processes may alter these findings.

While very idealized, these model results do show some similarities with the real ocean. The mean western boundary current follows the boundary all the way to the latitude of the interior gap in the ridge, even for very nonlinear flows, because of the influence of westward propagating Rossby waves. This may explain the abrupt eastward turn of the North Atlantic Current at the Northwest Corner, which is located at approximately the same latitude at the Charlie Gibbs Fracture Zone in the Mid-Atlantic Ridge. The northward turn and subsequent eastward flow of the Antarctic Circumpolar Current to the east of Drake Passage may be similarly influenced by the Kerguelen Plateau far to the east, as predicted by linear theory. The oscillation discussed here is characterized by nearly periodic shedding of anticyclonic vortices poleward of the outflow gap and a meridional shift of the separation latitude by several hundred $\mathrm{km}$. This type of variability is often seen near the separation point of the East Australian Current (Nilsson and Cresswell, 1981; Stanton, 1981). In addition, the separated Tasman Front exhibits a meandering pattern with maximum variability just off the western boundary and westward phase propagation, similar to the variability found here.

Acknowledgments. This study was supported by the National Science Foundation under Grants OCE-0826656, OCE-0959381 and OCE-1232389. Joe Pedlosky is thanked for many useful discussions. Hristina Hristova generously provided the complex EOF analysis scripts. Any opinions, findings, and conclusions or recommendations expressed in this material are those of the author and do not necessarily reflect the views of the National Science Foundation.

\section{REFERENCES}

Barnett, T. P. 1983. Interaction of the monsoon and Pacific trade wind system at interannual time scales, Part I: The equatorial zone. Mon. Wea. Rev., 111, 756-773.

Berloff, P. S. and J. C. McWilliams. 1999. Quasigeostrophic dynamics of the western boundary current. J. Phys. Oceanogr., 29, 2607-2634. 
Bower, A. S. and W. von Appen. 2008. Interannual variability in the pathways of the North Atlantic Current over the Mid-Atlantic Ridge and the impact of topography. J. Phys. Oceanogr., 38, 104-120.

Cessi, P., R. V. Condie and W. R. Young. 1990. Dissipative dynamics of western boundary currents. J. Mar. Res., 48, 677-700.

Dijkstra, H. A. and J. D. Neelin. 1995. On the attractors of an intermediate coupled ocean atmosphere model. Dyn. Atmos Ocean., 22, 19-48.

Dijkstra, H. A. and M. J. Molemaker. 1997. Symmetry breaking and overturning oscillations in thermohaline-driven flows. J. Fluid Mech., 331, 195-232.

Dijkstra, H. A. and M. Ghil. 2005. Low-frequency variability of the large-scale ocean circulation: a dynamical systems approach. Rev. Geophys., 43, RG3002, doi:10.1029/2002RG000122.

Ierley, G. and W. Young. 1991. Viscous instabilities in the western boundary layer. J. Phys. Oceanogr., $21,1323-1332$.

Jiang, S. F., F. Jin and M. Ghil. 1995. Multiple equilibria and aperiodic solutions in a wind driven double-gyre, shallow water model. J. Phys. Oceanogr., 25, 764-786.

Mulhearn, P. J. 1987. The Tasman Front: A study using satellite infrared imagery. J. Phys. Oceanogr., $17,1148-1155$.

LaCasce, J. H. and P. E. Isachsen. 2010. The linear models of the ACC. Prog. Oceanogr., 84, 139-157.

McCalpin, J. D. and D. B. Haidvogel. 1996. Phenomenology of the low-frequency variability in a reduced gravity quasi-geostrophic double-gyre model. J. Phys. Oceanogr., 26, 739-752.

Nilsson, C. S. and G. R. Cresswell, 1981. The formation and evolution of East Australian Current warm-core eddies. Prog. Oceanogr., 9, 133-183.

Pedlosky, J. 1998. Ocean Circulation Theory. Berlin: Springer-Verlag, pp. 43-47.

Sheremet, V. A., V. M. Kamenkovich and A. R. Pastushkov. 1995. Analysis of the barotropic model of the subtropical gyre in the ocean for finite Reynolds numbers, Part II. J. Mar. Res., 53, 995-1024.

Stanton, B. R. 1981. An oceanographic survey of the Tasman Front. New Zeal. J. Mar. Fresh., 15, 289-297.

Woityra, W. and T. Rossby. 2008. Current broadening as a mechanism for anticyclogenesis at the Northwest Corner of the North Atlantic Current. Geophys. Res. Let., 35, L05609, doi:10.1029/2007GL033063.

Zharkov, V. and D. Nof. 2008. Retroflection from slanted coastlines - circumventing the vorticity paradox. Ocean Sci., 4, 293-306.

Received: 30 October 2013; revised: 27 May 2014. 